\title{
PROGNOSTIC VALUE OF PD-L1 TUMOR EXPRESSION IN EGYPTIAN PATIENTS WITH EPITHELIAL BLADDER CANCER \\ ${ }^{1}$ Sherif Ahmed Abdel-Wahab, ${ }^{2}$ Manal Mohamed El Mahdy, ${ }^{1}$ Waleed Abdel-Monem Baioumy, ${ }^{1}$ Dina Ragab Diab, ${ }^{1}$ Sharehan Ibrahim Kotb
}

\author{
${ }^{1}$ Department of Clinical \\ Oncology and Nuclear \\ Medicine, and ${ }^{2}$ Department of \\ Pathology Faculty of Medicine, \\ Ain Shams University, Cairo, \\ Egypt. \\ Corresponding : \\ Sharehan Ibrahim Kotb \\ Mobile: 01003300693 \\ E mail: \\ hassansharehan@gmail.com \\ Received: 20/10/2019 \\ Accepted: 17/11/201
}

\begin{abstract}
:
Background: Bladder cancer ranks tenth in worldwide cancer incidence. With 550,000 newly diagnosed cases in 2018. It is the 7th most common cancer in men and the 17th most common cancer in women. In Egypt the incidence rate of cancer bladder in (2008-2011) $6.94 \%$ in both sexes and (10.7\%) among men.
\end{abstract}

Aim of the Work: To study the prognostic value of PD-L1 tissue tumoral expression in Egyptian patients with epithelial bladder cancer and its correlation with the different clinicopathological parameters.

Patients and Methods: This is a prospective, single arm, cross sectional study which was carried on newly diagnosed 30 patients with epithelial urinary bladder cancer were identified with follow up for 2 years from (Aug 2017-Sep 2019). At both Clinical Oncology and Nuclear Medicine department Ain Shams university Hospital and Nasser Institute Cancer Centre for researches and treatment (NICC).

Results: In our study we confirmed that high PD-L1 expression is correlated with prognosis, as patients with high PD-L1tumor expression cells had lower DFS and OS. The tumor PD-L1 over expression had high significant impact on PFS where patients who had PD-L1overexpression had mean 7.2 months compared to 11.4 months in patients who had low tumor PD-L1 expression ( $P$ value $=0.004$ ). also had significant impact on OS where patients who had high tumor PD-L1 over expression had mean OS 9.633 months compared to 12.00 months in patients who had low tumor PD-L1 expression, $(P$ value $=0.047)$.

Conclusion: Our study concluded that high expression of PDL1 was associated with low PFS and OS and worst prognosis.

Keywords: Urothelial bladder cancer, upper urinary urothelial carcinomas, overall survival, progression free survival

\section{INTRODUCTION}

Bladder cancer remains a challenge to treat, as evidenced by slowly declining yearly incidence rates, yet stable death rates for the last 15 years. Urothelial cancer is the fourth most common cause of cancer in men, with 74,000 new cases reported and 16,000 deaths in $2015^{(\mathbf{1})}$.
According to official statistics of the National Cancer Institute (Cairo University), bladder cancer accounts for $(6.9 \%)$, more in males than females in Egypt. Calculated age specific incidence rates for urinary bladder cancer in Egypt (2008-2011), progressive increase after the age of 50 years, to reach a sharp peak of crude rates in males $(12.6 / 100000)$, in females $(3.3 / 100.000)$ at the age group of 70-74 years ${ }^{(2)}$. 
Programmed death ligand-1 (PD-L1) is a $\mathrm{T}$-cell regulatory molecule that may be expressed on the surface of tumor and tumor-infiltrating immune cells. The PDL1/PD-1 pathway has been shown to be important in cancer progression ${ }^{(3)}$.

Tumor PD-L1 protein expression was found to be associated with a higher grade of tumors and a poorer survival rate in urothelial cancers ${ }^{(4)}$.

Only PD-L1 expression levels were found to be associated with an increased pathological stage and an independent predictor for all-cause mortality after cystectomy ${ }^{(\mathbf{5})}$.

Efforts have begun to delineate the role of the PD-L1 pathway in metastatic urothelial bladder cancer. Inhibition of the PD-L1 pathway by the investigational molecule, MPDL3280A, which blocks PD1/PD-L1 and B7.1/PD-L1 interactions, thereby completely inhibiting the PD-L1mediated immunosuppressive signals, has shown great clinical activity ${ }^{(6)}$.

Pembrolizumab In a phase I clinical trial of in patients with advanced bladder cancer patients whose tumors expressed $\geq 1 \%$ PD-L1, RR was $24 \%$, including three cases of $\mathrm{CR}^{(7)}$. Is approved in the secondline setting in 29 evaluable patients with advanced urothelial carcinoma (any location, 66\% metastatic) were reported at ASCO 2015. Overall response rate was $27.6 \%$ (11\% complete, $16 \%$ partial) with median duration of response not reached at 13 months median follow up ${ }^{(8)}$.

Atezolizumab (TECENTRIQ) (MPDL 3280A) another anti-PD-L1 antibody, yielded a RR of $26 \%$ in 68 patients with advanced bladder cancer ${ }^{(6)}$, it was approved for metastatic urothelial bladder cancer (UBC) progressing during or after platinumbased chemotherapy ${ }^{(9)}$.

Nivolumab (OPDIVO) (Bristol-Myers Squibb Company). On February 2, 2017, the U.S. Food and Drug Administration granted accelerated approval as a first line in treatment of patients with locally advanced or metastatic urothelial carcinoma who are ineligible for treatment with a platinum-containing chemotherapy ${ }^{(\mathbf{1 0})}$.

\section{AIM OF THE WORK}

The aim of this work is to study the prognostic value of PD-L1 tissue tumoral expression in Egyptian patients with epithelial bladder cancer and its correlation with the different clinicopathological parameters (e.g: tumor grade).

\section{PATIENTS AND METHODS}

This is a prospective, single arm, cross sectional study which was carried on newly diagnosed 30 patients with epithelial urinary bladder cancer were identified with follow up for 2 years from (Aug 2017-Sep 2019). At both Clinical Oncology and Nuclear Medicine department Ain Shams university Hospital and Nasser Institute Cancer Centre for researches and treatment (NICC).

\section{Study population:}

Inclusion criteria: Male and female patients with muscle invasive and metastatic epithelial bladder cancer, according to AJCC $7^{\text {th }}$ edition 2010. Performance status $<2$ (according to Eastern Cooperative Oncology Group (ECOG) ${ }^{(15)}$. Age range (18- 75) years old. Confirmed histological diagnosis of epithelial bladder cancer by TURBT. Written informed consent.

Exclusion Criteria: Prior or concurrent malignancy. Pregnant or lactating women. Non invasive bladder cancer. Performance status $>2$ according to ECOG. Comorbidities and poor renal profile.

Ethical Committee Approval: After taking the approval of research ethics committee (EC) of Faculty of medicine, Ain Shams University to perform this study, we got consent waiver as it is a prospective 
study, no intervention to be done on patients. Written permission to use human tumor tissues was obtained from patients.

Clinical evaluation: Data was retrieved from patients' medical records and included: Clinical evaluation including physical examination, blood tests (CBC, KFT and LFT), cystoscopy and TURBT, computed tomography (CT) chest, abdomen and pelvis, bone scan.

Pathological examination: Grading is according to The WHO 2016 system which is based on the WHO/ISUP 1998 classification and the WHO 2004 classification $^{(\mathbf{1 1})}$. Staging according to TNM staging of UBC according to $7^{\text {th }}$ edition of AJCC ${ }^{(12)}$.

Immunohistochemical staining: For immunohistochemistry staining, 4um thickness paraffin embedded tissue sections were prepared, and stained using bench mark ventana $(\mathrm{GX})$ automated immunostaner.

The staining protocol used was as follow: Loading slides, antibody and ultraview detection kit dispensers onto the BenchMark instrument. Selecting CC1 standard pretreatment. Antibody incubation set for 32 minutes at 37c. Start the run When the staining run is complete, more slides from instrument and rinse well with wash buffer. Cover slip. The primary antibody used B7-H1/PD-L1, polyclonal rabbit antibody, $0.1 \mathrm{ml}$ prediluted, novus biological, dilution 1:300.

Immunohistochemical evaluation: Immunohistochemistry (IHC), the paraffin blocks from all 30 patients were retrieved from the pathology unit at Ain Shams University Hospital and NICC Hospital.

Assessment of intratumoral and stromal TILs was done based on Immune-Oncology Biomarker Working Group guidelines and European Association of Urology. TIL scores were defined as the percentage of tumor area that was occupied by inflammatory (PD1 and PDL1) cells. TILs were scored as continuous variables with positivity cutoff set at $5 \%{ }^{(6,9 \& 3)}$.

The number of intratumoral and stromal lymphocytes was counted in three high power fields (magnification, $\mathrm{x} 400$ ). The counts were performed in areas of maximum lymphocytic infiltration. Foci demonstrating hemorrhage and/or necrosis were excluded.

Treatment: Surgery: 19/30 patients underwent surgery: Radical cystectomy (RC) was done in 17/19 while 2/19 patient had Partial cystectomy (PC). Chemotherapy: 10/30 patients were unfit for chemotherapy treatment and 20/30 patients received chemotherapy which was palliative in $1 / 20$ patients, adjuvant in $17 / 20$ patients, definitive CCRTH in $2 / 20$ patients. Gemcitabine/cisplatin combination chemotherapy was the most common regimen used in $12 / 20$ patients, paclitaxel/ carboplatin (5 AUC) was used in one patient. In patients who received concurrent chemoradiotherapy, weekly cisplatin was used in $2 / 20(10 \%)$ patients and carboplatin/gemcitabin were used in $5 / 20$ patients (25\%). Radiotherapy: 14/30 patients received radiotherapy in the form of definitive radiotherapy in $6 / 14$ patients, adjuvant radiotherapy in $6 / 14$ patients, $2 / 14$ patients received concurrent chemotherapy and radiotherapy. The technique of radiotherapy was 3D conformal in all patients.

\section{Objective of the study:}

Primary objective: To study the prognostic value of PD-L1 tissue tumoral expression in Egyptian patients with epithelial bladder cancer and its correlation with the different clinicopathological parameters.

Secondary objectives: To assess one to two years disease free survival (DFS) and overall survival (OS).

End points: Determination of epidemiological pattern of PDL-1 expression 
among Egyptian patients with muscle invasive and metastatic bladder cancer.

\section{Statistical analysis:}

Sample Size Justification:

Sample size was calculated using PASS $^{\circledR}$ version 11 program, setting the type-1 error $(\alpha)$ at 0.05 (95\% Confidence interval) and the power $(1-\beta)$ at 0.8 . Results from a previous study Nakanishi et al. ${ }^{(4)}$ showed that the mean PDL-1 score among grade 1,2 and 3 cases was $8.12 \pm 0.05$, $18.3 \pm 0.10$ and $26.1 \pm 0.08$ respectively. Calculation according to these values produced a minimal sample size of 20 cases $^{(14)}$.

\section{Data Management and Analysis:}

\section{RESULTS}

Table (1): Baseline patient characteristics
The collected data will be revised, coded, tabulated and introduced to a PC using Statistical package for Social Science (SPSS 15.0.1 for windows; SPSS Inc, Chicago, IL, 2001). Data will be presented as Mean and Standard deviation $( \pm$ SD) for quantitative parametric data, and Median and Interquartile range for quantitative nonparametric data. Frequency and percentage will be used for presenting qualitative data. Suitable analysis will be done according to the type of data obtained. Student T Test or Mann Whitney test will be used to analyze quantitative data while chi square test and fisher exact test will be used to analyze qualitative data. P- value: level of significance. $\mathrm{P}>0.05$ : Non significant $(\mathrm{NS})$. $\mathrm{P}<$ 0.05: Significant (S). $\mathrm{P}<0.01$ : Highly significant (HS).

\begin{tabular}{|c|c|c|}
\hline \multirow{5}{*}{ Age in years } & & No. $=30$ \\
\hline & Mean+/- SD & $61.53+/-8.94$ \\
\hline & Range & $42-75$ \\
\hline & $<60$ years & $15(50.0 \%)$ \\
\hline & $>60$ years & $15(50.0 \%)$ \\
\hline \multirow{2}{*}{ Gender } & Male & $22(73.3 \%)$ \\
\hline & Female & $8(26.7 \%)$ \\
\hline \multirow{2}{*}{ Smoking } & Yes & $21(70.0 \%)$ \\
\hline & No & $9(30.0 \%)$ \\
\hline \multirow[t]{3}{*}{ FH } & UB Carcinoma & $1(3.3 \%)$ \\
\hline & Other malignancies & $3(10 \%)$ \\
\hline & Negative & $26(86.6 \%)$ \\
\hline \multirow[t]{2}{*}{ ECOG } & I & $4(13.33 \%)$ \\
\hline & II & $26(86.66 \%)$ \\
\hline \multicolumn{3}{|l|}{ Presenting symptom } \\
\hline \multirow{2}{*}{ Heamaturia } & No & $8(26.6 \%)$ \\
\hline & Yes & $22(73.3 \%)$ \\
\hline \multirow{2}{*}{$\begin{array}{l}\text { Irritative bladder } \\
\text { symptoms }\end{array}$} & No & $24(80 \%)$ \\
\hline & Yes & $6(20 \%)$ \\
\hline \multirow{2}{*}{$\begin{array}{l}\text { Symptoms due to } \\
\text { metastasis }\end{array}$} & No & $29(96.6 \%)$ \\
\hline & Yes & $1(3.33 \%)$ \\
\hline \multirow{2}{*}{$\begin{array}{l}\text { Elevated kidney function } \\
\text { tests }\end{array}$} & No & $24(80 \%)$ \\
\hline & Yes & $6(20 \%)$ \\
\hline
\end{tabular}


Table (2): Tumor characterization among the studied patients

\begin{tabular}{|c|c|c|}
\hline & & Total no. $=30$ \\
\hline \multirow[t]{3}{*}{ Histopathology } & TCC & $20(66.7 \%)$ \\
\hline & $\mathrm{SCC}$ & $8(26.7 \%)$ \\
\hline & Others & $2(6.7 \%)$ \\
\hline \multirow[t]{3}{*}{ Grades } & & \\
\hline & Grade II & $9(30.0 \%)$ \\
\hline & Grade III & $21(70.0 \%)$ \\
\hline \multirow[t]{4}{*}{ N stage } & N0 & $6(20.0 \%)$ \\
\hline & N1 & $8(26.7 \%)$ \\
\hline & N2 & $6(20.0 \%)$ \\
\hline & $\mathrm{Nx}$ & $10(33.3 \%)$ \\
\hline \multirow[t]{5}{*}{ T stage } & & \\
\hline & $\mathrm{T} 2$ & $3(10.0 \%)$ \\
\hline & T3 & $12(40.0 \%)$ \\
\hline & $\mathrm{T} 4$ & $4(13.3 \%)$ \\
\hline & Unknown & $11(36.7 \%)$ \\
\hline \multirow[t]{2}{*}{ M stage } & M0 & $29(96.7 \%)$ \\
\hline & M1 & $1(3.3 \%)$ \\
\hline \multirow[t]{5}{*}{ TNM } & & \\
\hline & II & $2(6.7 \%)$ \\
\hline & III & $17(56.7 \%)$ \\
\hline & IV & $1(3.3 \%)$ \\
\hline & $\mathrm{Tx}$ & $10(33.3 \%)$ \\
\hline Method of staging & $\begin{array}{l}\text { Clinical } \\
\text { Pathological }\end{array}$ & $\begin{array}{l}11(36.7 \%) \\
19(63.3 \%)\end{array}$ \\
\hline \multirow[t]{2}{*}{ LVI } & Negative & $15(50.0 \%)$ \\
\hline & Positive & $15(50.0 \%)$ \\
\hline \multirow[t]{2}{*}{ PTNI } & Negative & $23(76.7 \%)$ \\
\hline & Positive & $7(23.3 \%)$ \\
\hline \multirow[t]{2}{*}{ Progression } & No & $19(63.3 \%)$ \\
\hline & Progressed & $11(36.7 \%)$ \\
\hline \multirow[t]{4}{*}{ PD-1 Immune cells (mean \%) } & Median (IQR) & $10(5-20)$ \\
\hline & Range & $1-30$ \\
\hline & $<10$ & $14(46.7 \%)$ \\
\hline & $>10$ & $16(53.3 \%)$ \\
\hline \multirow[t]{4}{*}{ Tumor PD-L1 over expression (\%) } & Median (IQR) & $25(10-50)$ \\
\hline & Range & $3-70$ \\
\hline & $<25$ & $15(50.0 \%)$ \\
\hline & $>25$ & $15(50.0 \%)$ \\
\hline
\end{tabular}

Table (3): $1^{\text {st }}$ line treatment.

\begin{tabular}{|l|l|c|c|}
\hline & & No. & $\%$ \\
\hline \multirow{3}{*}{ Surgery } & Yes & 19 & $63.4 \%$ \\
\cline { 2 - 4 } & No & 11 & $36.7 \%$ \\
\hline \multirow{5}{*}{ Type } & $\begin{array}{l}\text { Radical cystectomy, pelvic } \\
\text { lymphadenectomy }\end{array}$ & 17 & $56.7 \%$ \\
\cline { 2 - 4 } & $\begin{array}{l}\text { partial cystectomy without pelvic } \\
\text { lymphadenectomy }\end{array}$ & 2 & $6.7 \%$ \\
\hline \multirow{3}{*}{ Histological type } & TCC & 20 & $66.7 \%$ \\
\cline { 2 - 4 } & SCC & 8 & $26.7 \%$ \\
\hline \multirow{3}{*}{ Grade } & others & 2 & $6.7 \%$ \\
\cline { 2 - 4 } & GII & 9 & $30 \%$ \\
\cline { 2 - 4 } & GIII & 21 & $70 \%$ \\
\hline
\end{tabular}




\section{Sherif Ahmed Abdel-Wahab, et al.,}

\begin{tabular}{|c|c|c|c|}
\hline \multirow[t]{2}{*}{ Perivesical fat invasion } & Yes & 16 & $53.3 \%$ \\
\hline & No & 14 & $46.7 \%$ \\
\hline \multirow[t]{3}{*}{ Nearby organ invasion } & Uterus & 1 & $3.32 \%$ \\
\hline & Seminal vesicles & 1 & $3.32 \%$ \\
\hline & Prostate & 2 & $6.65 \%$ \\
\hline \multirow[t]{2}{*}{ Margins } & \multirow{2}{*}{$\begin{array}{l}\text { Infiltrated } \\
\text { Free }\end{array}$} & 4 & $13.3 \%$ \\
\hline & & 26 & $86.7 \%$ \\
\hline \multirow[t]{2}{*}{ LVI } & \multirow{2}{*}{$\begin{array}{l}\text { Yes } \\
\text { No }\end{array}$} & 15 & $50 \%$ \\
\hline & & 15 & $50 \%$ \\
\hline \multirow[b]{2}{*}{ PTNI } & \multirow{2}{*}{$\begin{array}{l}\text { Yes } \\
\text { No }\end{array}$} & 7 & $23.3 \%$ \\
\hline & & 23 & $76.7 \%$ \\
\hline \multirow[t]{5}{*}{ Pelvic LNs state } & \multirow{5}{*}{$\begin{array}{l}\text { No } \\
\text { N1 } \\
\text { N2 } \\
\text { Nx }\end{array}$} & No. & $\%$ \\
\hline & & 6 & $20 \%$ \\
\hline & & 8 & $26.7 \%$ \\
\hline & & 6 & $20 \%$ \\
\hline & & 10 & $33.3 \%$ \\
\hline \multirow[t]{3}{*}{ Chemotherapy } & \multirow{2}{*}{$\begin{array}{l}\text { Yes } \\
\text { No }\end{array}$} & 20 & $66.7 \%$ \\
\hline & & 10 & $33.3 \%$ \\
\hline & \multirow{2}{*}{$\begin{array}{l}\text { Adjuvant } \\
\text { Palliative } \\
\end{array}$} & 17 & $90 \%$ \\
\hline \multirow[t]{2}{*}{ Aim } & & 1 & $3.3 \%$ \\
\hline & Concurrent with radiotherapy & 2 & $6.7 \%$ \\
\hline \multirow[t]{4}{*}{ Type } & Gemzar/platinol & 12 & $60 \%$ \\
\hline & Taxol/platinol & 1 & $5 \%$ \\
\hline & carboplatin /gemzar & 5 & $25 \%$ \\
\hline & Weakly cisplatin in ccrth & 2 & $10 \%$ \\
\hline \multirow{5}{*}{$\begin{array}{l}\text { Toxicity (gem/platinol) } \\
\text { (No:11) }\end{array}$} & Neutropenia GII & 5 & $25 \%$ \\
\hline & Neutropenia GIII(gem/platinol) & 1 & $5 \%$ \\
\hline & Vomiting GI(gem/platinol) & 1 & $5 \%$ \\
\hline & Vomiting GII(gem/platinol) & 2 & $10 \%$ \\
\hline & Peripheral neuropathy GII(gem/platinol) & 2 & $10 \%$ \\
\hline \multirow[t]{2}{*}{ Radiotherapy } & Yes & 14 & $46.7 \%$ \\
\hline & No & 16 & $53.3 \%$ \\
\hline
\end{tabular}

Table (4): Kaplan Mayer analysis for PFS (months) among the studied patients

\begin{tabular}{|c|c|c|c|c|c|c|c|}
\hline \multirow{2}{*}{ No. } & \multicolumn{2}{|c|}{ PFS (months) } & \multicolumn{2}{|c|}{$95 \%$ CI } & \multicolumn{3}{c|}{ PFS (months) } \\
\cline { 2 - 7 } & Mean & SE & Lower & Upper & 3 months & 6 months & 12 months \\
\hline 30 & 9.3 & 0.674 & 7.98 & 10.62 & $86.70 \%$ & $70.00 \%$ & $63.30 \%$ \\
\hline
\end{tabular}

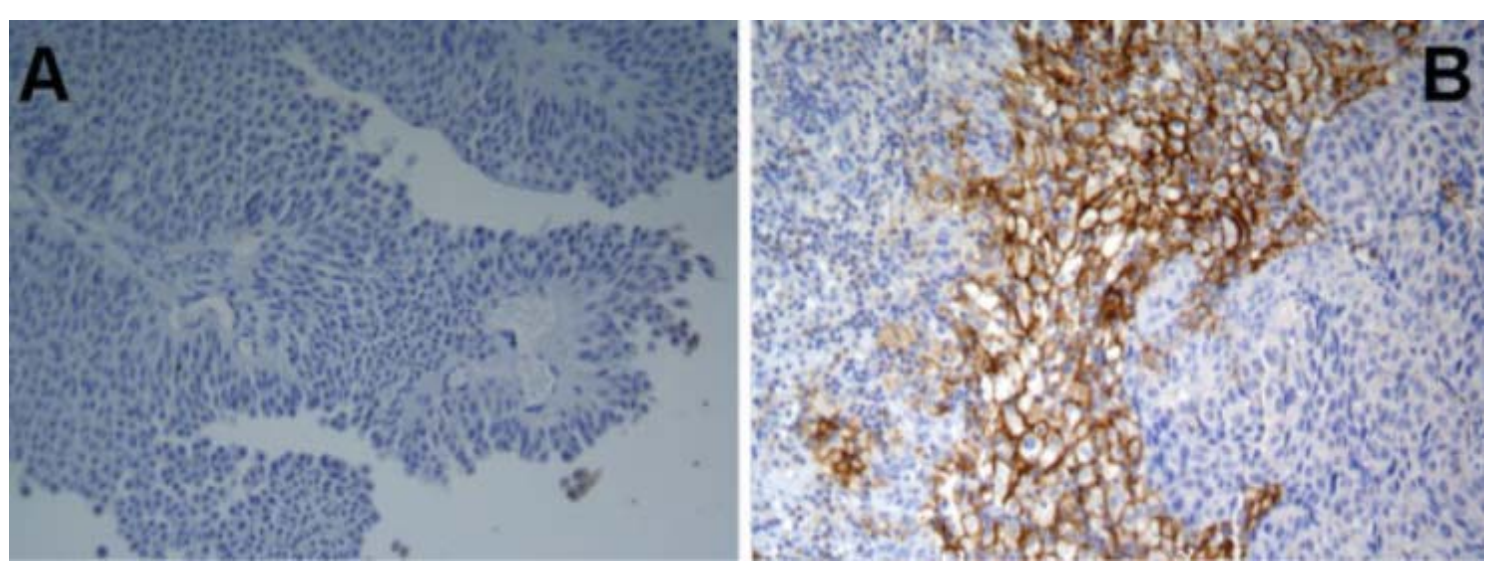

Fig (1): Immunohistostaining with anti PD-L1 mAb in urothelial cancer sections. Representative cases with low PD-L1 expression $<25 \%$ IQR (a), and high PD-L1 expression $>25 \%$ IQR (b). 
Table (5): Kaplan Mayer analysis for OS (months) among the studied patients

\begin{tabular}{|c|c|c|c|c|c|c|c|}
\hline \multirow{2}{*}{ No. } & \multicolumn{2}{|c|}{ OS (months) } & \multicolumn{2}{c|}{$95 \%$ CI } & \multicolumn{3}{c|}{ OS (months) } \\
\cline { 2 - 8 } & Mean & SE & Lower & Upper & 3 months & 6 months & 12 months \\
\hline 30 & 10.633 & 0.509 & 9.636 & 11.631 & $100.0 \%$ & $83.3 \%$ & $80.0 \%$ \\
\hline
\end{tabular}

Table (6): Correlation of PFS and OS (months) with PD1-immune cells and tumor PD-L1 overexpression

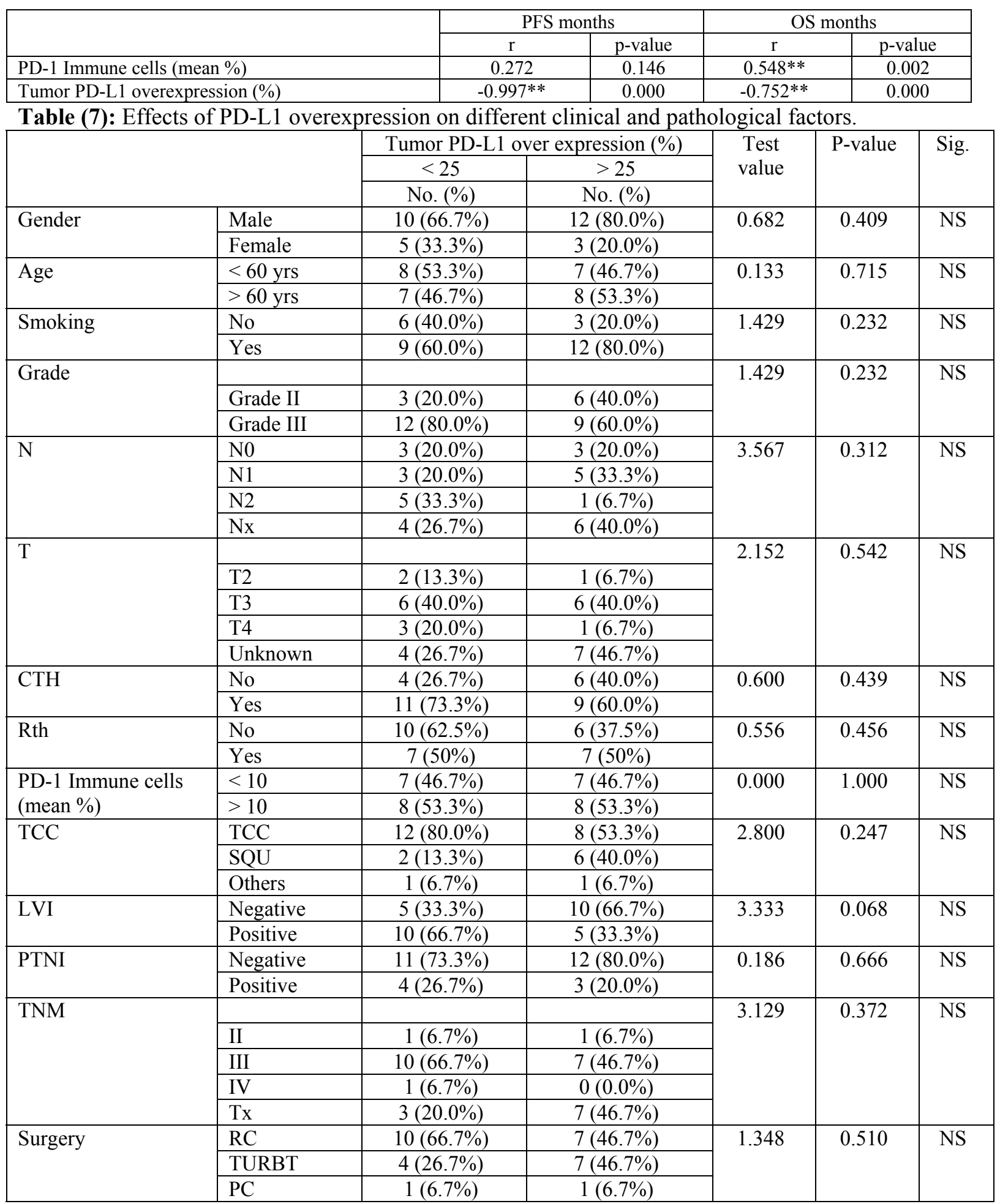




\section{DISCUSSION}

The Programmed death ligand-1(PD-L1) pathway is important in cancer immunesurveillance $^{(\mathbf{1 5})}$. And is an important target for cancer immunotherapy ${ }^{(\mathbf{1 6})}$. PD-L1 is a T-cell regulatory molecule that may be expressed on the surface of tumor and tumor-infiltrating immune cells. The PD-L1/PD-1 pathway has been shown to be important in bladder cancer $\operatorname{progression}^{(3)}$.

The mean patient age at diagnosis was 61.53 years (range 42 to 75 years), which is lower than the mean (IQR) age at diagnosis in other studies which was 66.4 years (range 60.3 to 67.0). Pichler et al, in a multicentre retrospective study which demonstrated that the mean (median, range) age of 67.8 (69, $36-84)$ years ${ }^{(17)}$.

In the present study, there was male predominance 22 males $(73.3 \%)$ and 8 females $(26.7 \%)$. Also in other studies there was male predominance including 48 men $(78.7 \%)$ and 13 women $(21.3 \%)$ Pichler et al. ${ }^{(17)}$ and by Brant et al, there was male predominance $81 \%$.

Smoking is the most important risk factor for the development of urothelial cancer, accounting for $50 \%$ of cases in men and $35 \%$ in women ${ }^{(\mathbf{1 8})}$. In our study, $70 \%$ of the patients were smokers.

The majority of the patients presented with hematuria (22 patients, $73.3 \%$ ) which agrees with almost all studies ${ }^{(19)}$.

The most common histological subtype in biopsied specimens was transitional cell carcinoma in 22 patients $(66.7 \%)$ which agrees with almost all studies ${ }^{\text {(20). This is }}$ comparable to that was found by Pichler et al in a multicentre retrospective study which demonstrated pure urothelial carcinoma in 48 patients $(78.8 \%)$ and subtypes of urothelial carcinoma in 13 patients $(21 \%)^{(17)}$.
In the present study 15 patients presented with MIBC (50\%) and 50\% of the patients presented with metastatic (distant metastases inlpatient (6.7\%) (bone metastasis) and lymph nodes metastases in 14 patients $(93.3 \%)$. This is comparable to that was found by Pichler et al in a multicentre retrospective study which demonstrated that 6 patients presented with NMIBC $(9.8 \%), 40$ patients presented with MIBC $(65.5 \%)$ and 15 patients presented with metastasis $(24.6 \%)^{(17)}$. In another study by Nakanishi et al, 35 patients presented with NMIBC (53.8\%), 23 patients presented with MIBC (35\%) and 7 patients presented with metastasis $(10.7 \%)^{(\mathbf{4})}$.

In the present study 15 patients $(50 \%)$ had LVI (lymphovascular invasion), and 7 patients $(23.3 \%)$ had PTNI (peritumoral neural invasion). In another study by Pichler et al, 33 patients had LVI positive $(62.3 \%)$ and 23 patients had LVI negative $(37.7 \%)^{(\mathbf{1 7 )}}$. In another study by Brant et al, 28 patients had negative LVI (10\%), positive focal LVI in 90 patients $(32 \%)$ and positive diffuse LVI in 162 patients $(58 \%)$.

In the present study 19 patients $(63.4 \%)$ underwent radical surgery, 17 of them underwent radical cystectomy with pelvic lymphadenectomy (92.8\%), and partial cystectomy without pelvic lymphadenectomy was done in 2 patients $(7.2 \%)$. In a retrospective study including 65 patients by Nakanishi et al, 30 patients (46.15\%) underwent transurethral resection of bladder tumor, 20 patients (30.7\%) underwent radical cystectomy for a bladder tumor; and 15 patients(23\%) underwent radical nephroureterectomy for ureter or renal pelvic tumors ${ }^{(4)}$.

In our population study, 10 patients $(33.3 \%)$ were unfit for chemotherapy treatment and 20 patients $(66.7 \%)$ received chemotherapy which was palliative in 1 patient $(5 \%)$, adjuvant in 17 patients $(85 \%)$, definitive CCRTH in 2 patients $(10 \%)$. Gemcitabine/cisplatin combination chemo 
therapy was the most common regimen used in 12 patients $(60 \%)$, paclitaxel /carboplatin (5 AUC) was used in one patient $(5 \%)$. In patients who received concurrent chemoradiotherapy, weekly cisplatin was used in 2patients (10\%) and carboplatin/gemcitabin were used in 5patients $(25 \%)$.In a retrospective study, patients who experienced disease recurrence after RC (radical cystectomy) and were then consequently treated with platinum-based, first-line chemotherapy: 33patients $(54.1 \%)$ were cisplatin-fit and received gemcitabine/ cisplatin and 28patients (45.9\%) underwent gemcitabine/ carboplatin chemotherapy ${ }^{(\mathbf{1 7})}$.

In the present study 7 patients in CR $23.3 \%$ (complete response), 12 patients in SD $40 \%$ (stationary disease) and 11 patients $36.7 \%$ required second line treatment due to progression after first line treatment, 10 progressive disease patients $(90 \%)$ received chemotherapy and one patient $(10 \%)$ received radiotherapy (palliative RTh of bone). The treatment response was PD (progressive disease) in 7 patients $(63.6 \%)$, while 4 patients $(36.3)$ had SD (stationary disease). Progression occurred in the bone in 2 patients $(28.6 \%)$, 3 patients $(42.8 \%)$ in liver, peritoneal metastasis in 2 patients $(28.6 \%)$.In another study, the median follow-up for all patients was 26 months, with a range of 1-118 months. Nine patients $(13.8 \%)$ died of urothelial cancer and another $2(3.1 \%)$ died of other causes (pulmonary embolism and sepsis $)^{(\mathbf{4})}$.

We demonstrated that the mean PFS was 9.3 months. The 1 year PFS was $63.30 \%$, In another study, the median recurrence-free survival from RC (radical cystectomy) was 9.8 months (95\% CI 7.212.5) in all patients, with a median DSS (disease specific survival) from recurrence of 9.9 months $(95 \% \text { CI } 6.2-13.7)^{(17)}$.

The mean OS was 10.633 months and the 1 year OS is $80.0 \%$.The 5 year OS was not reached at the end of the follow-up. In another study the median OS after RC was confirmed with 25.5 months (95\% CI 10.1 40.8) in all patients ${ }^{(\mathbf{1 7})}$. This difference can be attributed to that our patients presented with more advanced stages and also most of them lost follow up resulting in lack of survival data in our study.

The predictive value of PD-L1 is limited due to a lack of uniform definition of PD-L1 positivity, a lack of standard assays for PD-L1 expression Mukherji et al. ${ }^{(21)}$, with different semi-quantitative scores of the PD-L1 expression status in clinical trials, different PD-L1 antibody clones, and thus resulting in heterogeneous data $^{(10)}$.

We examined the prognostic value of TILs (PD-L1 and PD-1) in pretreatment biopsies of urothelial bladder cancer (MIBC and metastatic disease) and the cutoff point $(>=5 \%)$ for the actual PD-L1 expression, also by Skala et al. had examined the prognostic and predictive role of TILs in baseline, pretreatment biopsies of urothelial bladder cancer regarding its significant role and the cutoff point $(>=5 \%)$ for the actual PD-L1 expression and differentiation between high and low TILs ${ }^{(13)}$.

Nakanishi et al. Reviewed 65 patients in a study in Kumamoto university medical hospital, the expression of B7-H1 (PD-L1) was demonstrated on the plasma membrane and the cytoplasm of cancer cells in a focal pattern, the mean percentage of B7-H1 positive cells among the cancer cells was $21.1 \pm 11.0 \%$, median $20 \%$, range (2.1-47. $1 \%$ ). Where TILs were classified into 2 categories based on the cutoff point, which was $12.2 \%$. This value was the mean of the median percentage of $\mathrm{B} 7-\mathrm{H} 1$ expression together with WHO grade $1(6.8 \%)$ and grade $2(17.5 \%)$ cases $^{(4)}$. In another study by Brant et al, the tumor was considered positive for PD-L1 if $\geq 1 \%$ of tumor cells and had histological evidence of plasma membrane staining ${ }^{(\mathbf{1 3})}$. 
We were searching for the correlation between the TILs (PD-L1 and PD-1) overexpression and the prognosis in epithelial bladder cancer cases (MIBC and metastatic), where any increase in PDL1expression level will worsen the prognosis, also will be translated to lower DFS (disease free survival).

We classified TILs as a continuous parameter and into 2 groups high and low expression groups, based on the cutoff point which was $(\geq 5 \%)$ and the median interquartile rang (IQR) of intratumoral and stromal TILs which was in PD-L1 25\% [10\%-50\%].The median IQR of PD-L1 higher than $25 \%$ had worse impact on PFS and OS. The median IQR of PD-1 was $10 \%$ (5\%-20\%) with insignificant results regarding prognosis. Huang et al. had shown that the expression of PD-L1, B7.1 and PD-1 was associated with more aggressive clinicopathological parameters of bladder cancer in three independent cancer datasets. They were identified from the Gene Expression Omnibus database (GEO), with a sample size $>=100$ patients. The TILs expression levels were divided into high and low levels using the higher quartile expression level as the cut-off point for the Kaplan-Meier survival analysis ${ }^{(\mathbf{2 2})}$.

In comparison to another study by Pichler et al. PD-L1 expression on TCs (tumor cells) was defined by the percentage of PD-L1 positive tumor cells $(<1 \%=\mathrm{IC} 0$, $\geq 1 \%$ but $<5 \%=\mathrm{IC} 1, \geq 5 \%=\mathrm{IC} 2 / 3)$, and was considered negative or positive for ICs (immune cells) $^{(\mathbf{1 7})}$.

In the present study, the age had insignificant impact on PD-L1 expression where 15 patients who had $>25 \%$ IQR tumor PD-L1 over expression and 15 patients who had $<25 \%$ IQR tumor PD-L1 over expression $(\mathrm{P}$ value $=0.715)$. Also in another study by Pichler et al, insignificant correlation between patient age and PD-L1 expression, $\mathrm{P}$ value $=0.934$ Pichler et al. ${ }^{(17)}$ and insignificant by Brant et al, as $\mathrm{P}$ value $=$ 0.705 .

We observed no association between PD-L1 expression and patient gender, where 12 males had $>25 \%$ IQR tumor PDLloverexpression and 3 females had $>25 \%$ IQR tumor PD-L1 over expression, P value $=0.409$. Pichler et al. reviewed a nonsignificant association between B7-H1 (PD$\mathrm{L} 1)$ expression and patient gender. $\mathrm{P}$ value $=0.757$ Pichler et al. ${ }^{(17)}$ and also by Brant et al, $\mathrm{P}$ value $=0.529$, and insignificant by Nakanishi et al, $\mathrm{P}=0.994^{(4)}$.

Smoking was found to have insignificant impact on PD-L1 expression where 12 smoker patients who had $>25 \%$ IQR tumor PD-Lloverexpression and 3 non smokers who had $>25 \%$ IQR tumor PD-L1 over expression, $\mathrm{P}$ value 0.232.Also in multistudies analysis by Xaingli et al, smoking history had insignificant impact with PDL-1 expression $(\mathrm{n}=3, \mathrm{OR}=1.15$, 95\% CI: 0.65-2.04; $p=0.902)^{(23)}$.

Grading was found to have insignificant impact on PD-L1 expression where 6 patients with $\mathrm{G} 2$ had $>25 \%$ IQR tumor PD-L1 over expression and 9 patients with G3 had $>25 \%$ IQR tumor PD-L1 over expression, $\mathrm{P}$ value $=0.232$. In comparison to Nakanishi et al and Huang et al, showed a significant association between B7-H1 expression and WHO grade $(\mathrm{p}<0.001)^{(\mathbf{4 , 2 2})}$.

T stage (tumor size) was found to have insignificant impact on PD-L1 expression, ( $\mathrm{P}$ value $=0.542)$. ( $\mathbf{N}$ stage) was found to have insignificant impact on PD-L1 expression, $(P$ value $=0.312)$. Staging had insignificant impact on PD-L1 expression, $(\mathrm{P}=0.372)$. In comparison to Nakanish et al, who found tumor associated B7-H1 expression was associated significantly $(\mathrm{P}=0.031)$ with primary tumor $(\mathrm{T})$ classification but no significant association was found between B7-H1 expression and either primary node (N) $\mathrm{P}$ value $=0.376$ or primary stage classification, $\mathrm{P}$ value $=0.084^{(4)}$. 
In meta-analysis of 11 relevant studies on tumor stage demonstrated that the tumor stage was not significantly associated with PD-L1 expression on tumor-infiltrating ICs in patients with bladder cancer. $(n=4$, $\mathrm{OR}=1.43$, 95\% CI: $0.93-2.21 ; p=0.211)^{(\mathbf{2 3})}$.

In comparison to Huang et al, reviewed that the expression levels of PD-L1 were significantly higher with a higher $\mathrm{T}$ stage $(\mathrm{p}<0.001)$ and were also significantly higher with a higher $\mathrm{N}$ stage of the tumors $(p<0.001)$.In addition, the mRNA expression of PD-L1 was significantly higher $(p=0.039)$ in primary tumors with M1 ( $\mathrm{n}=7)$ stage than those with M0 stage $(\mathrm{n}=158)^{(22)}$.

Prior use of chemotherapy was found to have insignificant impact on PD-L1 expression $(\mathrm{P}$ value $=0.439)$. Also in multi studies analysis by Xaingli et al, showed insignificant relation between PD-L1 expression on bladder cancer tumor cells and prior use of chemotherapy $(n=4$, $\mathrm{OR}=0.72 ， \quad 95 \% \quad \mathrm{CI}: \quad 0.31-1.69 ; p=0.066$; random-effects) ${ }^{(23)}$.

We found that a high level of expression of PD-L1 was associated with a shorter survival time suggesting that PD-L1 is a potential prognostic factor and a promising therapeutic target in bladder cancer.PD-1 was expressed at high levels on TILs but had insignificant relation with PFS and OS.

The tumor PD-L1 over expression had significant impact on OS where patients who had $>25 \%$ IQR tumor PD-L1 over expression had mean 9.633 months compared to 12.00 months in patients who had $<25 \%$ IQR tumor PD-L1 over expression, $(\mathrm{P}$ value $=0.047)$. Correlation between tumor PD-L1 tumor over expression and PFS and OS, we found indirect relation between them (P value $=0.000$ ), so the more tumor PD-L1 over expression the worst prognosis as decreased PFS and OS. The same was found by Nakanishi et al, Reviewed that B7-H1(PD-L1) expression associated with prognosis, overall survival was worse in patients with high B7-H1 tumor expression (high B7-H1 group) 5-year survival was 55 $\%$ vs $100 \%, \quad(p=0.021)$. PFS was also significant worse in the (high B7-H1 group) than in the (lowB7-H1 group), 5-year survival $61 \%$ vs $100 \%(p=0.04)^{(4)}$.

Huang et al. concluded that a high level of expression of PD-L1 was associated with a shorter survival time. In GSE13507, patients with bladder cancer expressing high levels of PD-L1 and B7.1 had a mean overall survival of 62 months compared to 115 months for those with bladder cancer expressing low levels of PD-L1 and B7.1 $(p=0.001)$. similar results were obtained in GSE32894, patients with bladder cancer expressing high level of the two genes had a mean survival of 79 months compared to 100 months for those patients with their bladder cancers expressing low levels of the two genes $(p=0.021)$. Suggesting that PD$\mathrm{L} 1$ is a potential prognostic factor and a promising therapeutic target in bladder cancer $^{(22)}$.

These findings are in accordance with those of Boorjian et al who reported that PD-L1 over expression on TCs was associated with all-cause mortality in 167 bladder cancer patients with organ-confined disease treated with RC (radical cystectomy) ${ }^{(\mathbf{5})}$.

Zhu et al. ${ }^{(23)}$, in multi analysis studies suggested that positive PD-L1 expression on TCs (tumor cells) in patients with Bladder Cancer could be considered a significant biomarker for diagnosis of advanced stage disease.

We found the immune cells PD-1 over expression had insignificant impact on PFS where patients who had $>10 \%$ IQR immune PD-1 over expression in 16 patients $(53.3 \%)$ had mean 9.438 months compared to 9.143 months in patients who had $<10 \%$ IQR 
immune PD-1 over expression 14 patients $(46.6 \%)$, ( $P$ value $=0.978)$. Also had insignificant impact on OS where patients who had $>10 \%$ IQR immune PD-1 over expression had mean 11.125 months compared to 10.071 months in patients who had $<10 \%$ IQR immune PD-1 over expression, (P value $=0.286)$. The same was found by Pichler et al, the PD-1 expression on ICs (immune cells) of primary tumors and metastatic sites had no significant influence on DSS (median DSS, positive vs. negative: 10.5 vs. 8.5 months [primary tumor], $p=$ $0.109 ; 26.9$ vs. 8.9 months [metastatic site], $p=0.127)^{(\mathbf{1 7 )}}$.

Nakanishi et al, Reviewed that PD-1 was expressed at high levels on major populations of both CD4, CD8 TILs. The mean percentage of CD4 and CD8 TILs expressing PD-1 were $86.0+/-18.5 \%$ and $80.6 \pm 14.0 \%$ respectively. PD-1 expression was significant associated $(\mathrm{p}=0.009)$ between CD4 and CD8 TILs and an inverse association was observed between CD4/ CD8 ratio and $\mathrm{PD}-1$ expression on CD8 TILs $(p=0.005)$, this indicated a large number of the CD8 $\mathrm{T}$ cells infiltrating into the urothelial cancer cells expressing PD-1. Thus it is possible that the induced B7-H1 on urothelial cancer cells interacts with PD1 on TILs and that this interaction regulates the effect or function of TILs resulting in tumor progression ${ }^{(4)}$.

Huang et al, found the expressions of PD-L1, B7.1 and PD-1 were significantly correlated with each other. Only the PD-L1 and B7.1 co-over expression was significantly associated with a shorter survival time in bladder cancer patients ${ }^{(\mathbf{2 2})}$.

\section{Conclusion:}

The study also supported the hypothesis that positive PD-L1 expression based on staining different cellular populations (tumor cells, tumor-infiltrating ICs, or both) associated with worst prognosis and might be associated with improved response to
PD-1/PD-L1 inhibitors in advanced UC (urinary bladder cancer) patients.

\section{REFERENCES}

1. Siegel R, Miller K, Jemal A, et al. (2015): Cancer Statistics, CA Cancer J Clin; 65: 529.

2. Fedewa SA, Soliman AS, Ismail K, et al. (2009): Incidence analyses of bladder cancer in the Nile delta region of Egypt. Cancer Epidemiology, vol. 33, no. 3-4, pp. 176-181.

3. Chen DS, Irving BA and Hodi FS (2012): Olecular pathways: Next-generation immunotherapy - inhibiting programmed death-ligand 1 and programmed death-1. Clin Cancer Res; 18: 6580-6587.

4. Nakanishi J, Wada Y, Matsumoto K, et al. (2007): Over expression of B7-H1 (PDL1) significantly associates with tumor grade and postoperative prognosis in human urothelial cancers. Cancer Immunol Immunother; 56:1173-82.

5. Boorjian SA, Sheinin Y, Crispen PL, Farmer SA, Lohse CM, Kuntz SM, Leibovich BC, et al. (2008): T-cell coregulatory molecule expression in urothelial cell carcinoma: clinicopathologic correlateions and association with survival. Clin Cancer Res; 14(15):4800-8.

6. Powles T, Eder JP, Fine GD, et al. (2014): MPDL3280A (anti-PD-L1) treatment leads to clinical activity in metastatic bladder cancer. Nature; 515: 558-562.

7. Plimack ER, Gupta S, Bellmunt J, et al. (2014): Phase 1B study of pembrolizumab (pembro; MK-3475) in patients (pts) with advanced urothelial tract cancer. Ann Oncol (Meeting Abstracts) 25: LBA23.

8. Plimack ER, Bellmunt J, Gupta S, Berger $\mathrm{R}$, et al. (2015): Pembrolizumab (MK3475) for advanced urothelial cancer: Updated results and biomarker analysis from KEYNOTE-012. Chicago, IL: American Society of Clinical Oncology. Abstr 4502.

9. Rosenberg JE, Hoffman-Censits J, Powles T, et al. (2016): Atezolizumab in patients with locally advanced and metastatic 
urothelial carcinoma who have progressed following treatment with platinum-based chemotherapy: A single-arm, multicentre, phase 2 trial. Lancet; 387: 1909-1920.

10. Sharma P, Retz M, Siefker-Radtke A, et al. (2017): Nivolumab in metastatic urothelial carcinoma after platinum therapy (CheckMate 275): a multicentre, singlearm, phase 2 trial. Lancet Oncol; 18:312322.

11. Moch H, Humphrey PA, Ulbright TM, et al. (2016): WHO classification of tumours of the urinary system and male genital organs. Geneva, Switserland.

12. Edge SB, Byrd DR, Compton CC, et al. (2010): AJCC Cancer Staging Manual. 7th ed. New York, NY: Springer; 573-574.

13. Skala SL, Liu TY, Udager AM, Weizer AZ, Montgomery JS, Palapattu GS, et al. (2017): Programmed Death-Ligand 1 Expression in upper tract urothelial carcinoma. Eur Urol Focus; 3(4-5): 502509.

14. Hintze J (2011): PASS 11. NCSS, LLC. Kaysville, Utah, USA. Overexpression of B7-H1 (PD-L1) significantly associates with tumor grade and postoperative prognosis in human urothelial cancers.

15. Corthay A (2014): Does the immune system naturally protect against cancer? Front Immunol 5: 197.

16. Kyi C and Postow MA (2014): Checkpoint blocking antibodies in cancer immunetherapy. FEBS Lett; 588: 368-376.

17. Pichler R, Heidegger I, Fritz J, Danzl M, Sprung S, Zelger B, Brunner A and Pircher A, et al. (2017): PD-L1 expression in bladder cancer and metastasis and its influence on oncologic outcome after cystectomy. Oncotarget; 8(40): 6684966864.

18. Zeegers MP, Tan FE, Dorant E, Brandt PA, et al. (2000): The impact of characteristics of cigarette smoking on urinary tract cancer risk: a meta-analysis of epidemiologic studies. Cancer; 89(3): 630-639.

19. Hall MC, Chang SS, Dalbagni G, et al. (2007): Guideline for the management of nonmuscle invasive bladder cancer (stages Ta, T1, and Tis): update. J Urol; 178(6): 2314-2330.

20. Eble JN, Sauter G, Epstein JI, et al. (2004): Pathology and Genetics of Tumours of the Urinary System and Male Genital Organs. IARC Press; Lyon: World Health Organization Classification of Tumours; $p$. 359.

21. Mukherji D, Jabbour MN, Saroufim M, Temraz S, Nasr R, Charafeddine M, Assi $\mathrm{R}$, Shamseddine A, Tawil AN, et al. (2015): Programmed Death-Ligand 1 Expression in Muscle- Invasive Bladder Cancer Cystectomy Specimens and Lymph Node Metastasis: A Reliable Treatment Selection Biomarker? Clin Genitourin Cancer; 14: 183-7.

22. Huang Y, Zhang SD, Mccrudden C, Chan KW, Lin Y and Kwok HF, et al. (2015): The prognostic significance of PD-L1 in bladder cancer, DOI: $10.3892 /$ or. 2015. 3933.

23. Zhu L, Sun J, Wang L, et al. (2019): Prognostic and clinicopathological significance of PD-L1 in patients with bladder cancer: a meta-analysis. Frontiers in Pharmacology, 10: 962. 


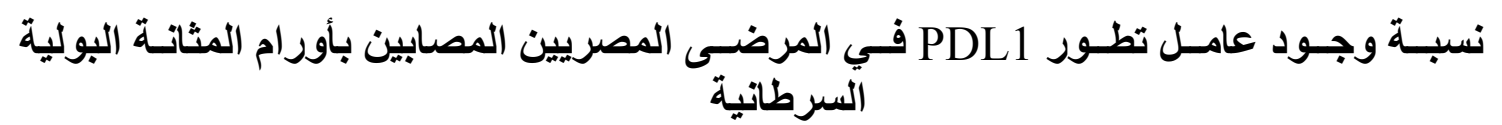

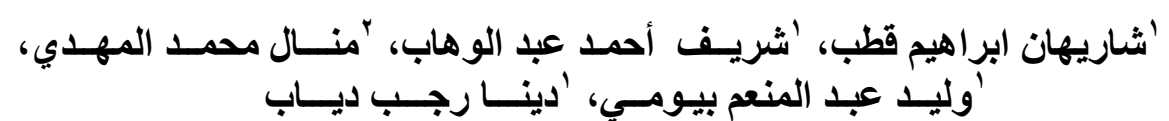

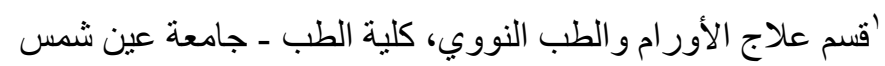

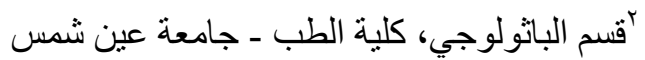

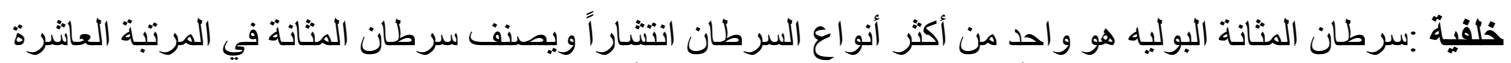

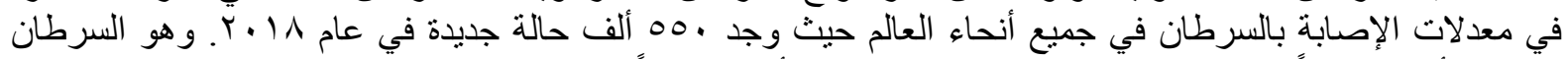

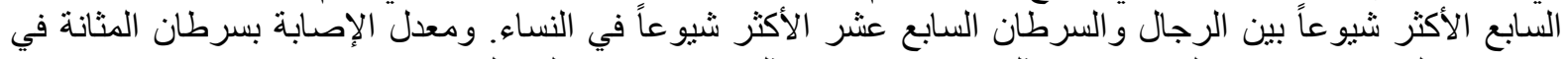

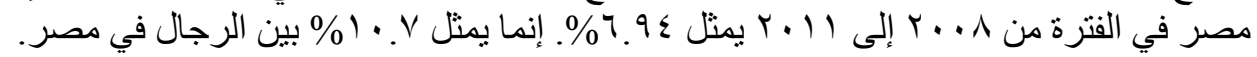

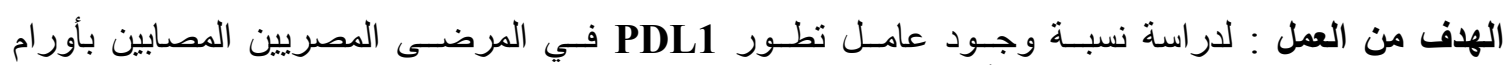

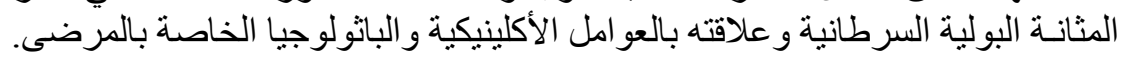

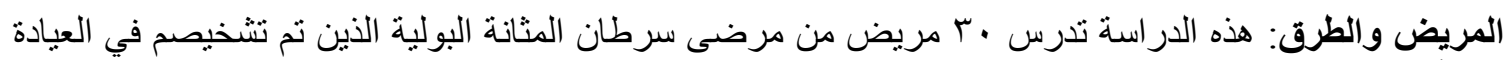

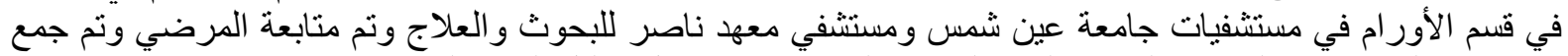
خصائص المرضي، وام في الحالات الصحية لهم و البيانات المرضية من خلال تحليل الرسم البياني.

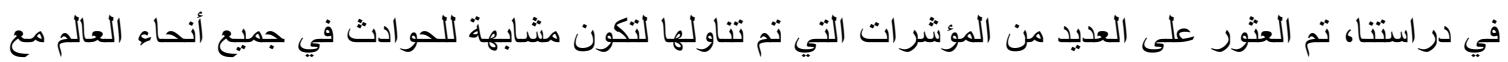
وجود بعض الاختلافات.

النتائج :أنه كلماز ادت نسبة الخلايا المناعية كلما أثر ذلك علي درجة تطور المرض و لا توجد حتي الآن نسبة محددة يمكن وضعها كقيمة ثابتة.

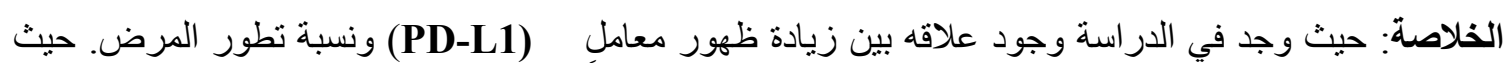

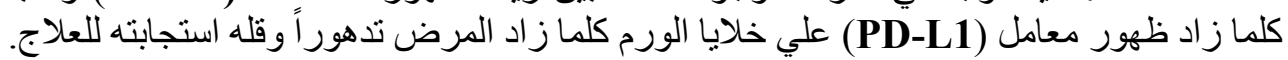

